\title{
Colite Cística Profunda. Relato de Caso
}

\author{
Colitis Cystica Profunda. Case Reporte
}

\author{
FELIPE FELIICIO $^{1}$; JOSÉ MAURO DOS SANTOS ${ }^{1} ;$ JOÃO CARLOS COSTA DE OLIVEIRA $^{1}$; \\ HUMBERTOFENNER LYRAJUNIOR ${ }^{1}$; ILARIO FROEHNER JUNIOR ${ }^{2}$; SERGIOCAMPOS MELLO JR ${ }^{3}$
}

${ }^{1}$ Serviço de Coloproctologia do Hospital Universitário da Universidade Federal de Santa Catarina (HU - UFSC);

${ }^{2}$ Residente de Cirurgia Geral do HU -UFSC; ${ }^{3}$ Residente de Cirurgia do Aparelho Digestivo do HU - UFSC.

FELÍCIO F; SANTOS JM; OLIVEIRA JCC; LYRA JUNIOR HF; FROEHNER JUNIOR I; MELLO JR SC. Colite Cística Profunda. Relato de Caso. Rev bras Coloproct, 2009;29(3): 377-381.

RESUMO: A colite cística profunda consiste na presença de cistos submucosos, contendo muco, principalmente no reto e no cólon esquerdo. De etiologia controversa, com pouco mais de 200 casos relatados na literatura mundial. Tem importância pela capacidade de mimetizar neoplasia maligna colorretal. Descreveremos um caso de colite cística profunda localizada no reto e submetida a tratamento cirúrgico, seu acompanhamento pós-operatório e revisão da literatura.

Descritores: Colite, cistos, muco, hemorragia gastrointestinal, reto.

\section{INTRODUÇÃO}

Colite cística profunda é doença colorretal benigna e rara, tendo sido descritos pouco mais de 200 casos na literatura médica indexada ${ }^{(1)}$.

Caracteriza-se microscopicamente pela presença de cistos submucosos repletos de muco e apresenta-se macroscopicamente sob a forma de pólipos sésseis e lesões elevadas com mucosa suprajacente edemaciada podendo apresentar ulcerações ${ }^{(1,2,4)}$. Localizadas isoladamente no reto em mais de $70 \%$ dos casos, associadas ao cólon esquerdo em $14,58 \%$, podendo acometer difusamente o intestino grosso ${ }^{(8)}$.

De forma geral, não apresenta preferência por gênero, com ampla faixa etária de incidência, predominando em pacientes entre a terceira e quarta décadas ${ }^{(7)}$, pode estar presente desde os primeiros anos de vida, já relatado caso em paciente de 04 anos ${ }^{(4)}$.

Suas manifestações clínicas variam de lesões assintomáticas a quadros de sangramento retal (68\%), eliminação de muco (43\%), tenesmo (13\%) e dor abdominal $(12 \%)^{(4)}$.
A colite cística profunda ganha importância por suas características clínicas e morfológicas, mimetizando a neoplasia maligna colorretal ${ }^{(5)}$.

\section{RELATO DE CASO}

Paciente masculino, 25 anos, mecânico industrial, previamente hígido, comparece ao ambulatório de Coloproctologia do Hospital Universitário da Universidade Federal de Santa Catarina queixando-se de hematoquezia há 01 ano associada a mucorreia esporádica. Sem outras queixas ou comorbidades.

Traz retossigmoidoscopia e colonoscopia já realizadas em sua cidade (Figura 1), que descrevem 02 lesões polipóides sésseis, de superfície lisa, coloração rósea, com cerca de $04 \mathrm{~cm}$ de diâmetro localizadas a $05 \mathrm{~cm}$ da margem anal, onde foram tomadas biópsias e cujo exame anátomo-patológico concluiu ser um adenoma viloso com atipias discretas (Figura 2).

No exame proctológico havia plicomas anais à inspeção e ao toque retal lesão elevada, endurecida, bem delimitada, indolor, fixa à parede retal ântero-late-

Trabalho realizado no Serviço de Coloproctologia do Hospital Universitário da Universidade Federal de Santa Catarina (HU - UFSC).

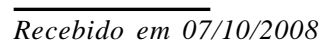

Aceito para publicação em 03/11/2008 
ral direita, iniciando a $02 \mathrm{~cm}$ e estendendo-se até $07 \mathrm{~cm}$ da margem anal.

Diante desse exame proctológico e resultado histopatológico indicamos ressecção local da lesão, por via transanal. $\mathrm{O}$ paciente foi internado às vésperas do procedimento para preparo de cólon e submetido a ressecção local com sutura primária (Figuras 3 e 4). Evolui no primeiro dia pós-operatório com enterorragia volumosa necessitando de reintervenção cirúrgica e hemostasia de um vaso com sangramento ativo na ferida. Teve alta em ótimo estado no sétimo dia de pósoperatório. É acompanhado no ambulatório de coloproctologia há 08 meses assintomático e sem recidiva endoscópica.

A análise macroscópica da peça mostrou área fibroadiposa de 3,0 x 3,5 x 0,5 cm; aos cortes, espessura máxima da parede de $1,5 \mathrm{~cm}$. Na microscopia foram achados espaços císticos preenchidos por muco, sem componentes neoplásicos, caracterizando-se histologicamente como colite cística profunda (Figura 5).

\section{DISCUSSÃO}

O primeiro relato da colite cística profunda data de 1766, quando Stark descreveu dois casos em pacientes com disenteria crônica ${ }^{(3)}$. Virchow, em 1863, estabeleceu o termo "colitis cystica polyposa" ao citar um caso de cistos submucosos polipóides ${ }^{(1)}$.

Apenas em 1957, a nomenclatura colite cística profunda foi usada, com a finalidade de diferenciar da colite cística superficial (presença de cistos na mucosa em pacientes com pelagra) ${ }^{(4)}$.

Diversos termos têm sido usados como sinônimos à colite cística profunda, entre eles: úlcera retal solitária, síndrome do prolapso retal, invasão pseudocarcinomatosa, cistos enterógenos do reto, pólipos hamartomatosos invertidos do reto e mucocele intestinal ${ }^{(7)}$.

Com incidência similar entre os pacientes masculinos e femininos, a faixa etária varia amplamente incluindo os primeiros anos de vida, concentrando-se na terceira e quarta décadas. Um estudo retrospectivo de 144 casos descreveu pacientes de 4 a 76 anos de idade $^{(4)}$.

Herman e Nabseth (1973) classificaram a colite cística profunda em três formas, de acordo com sua distribuição: difusa, segmentar e localizada. $\mathrm{Na}$ forma difusa, encontram-se lesões em todo o cólon,

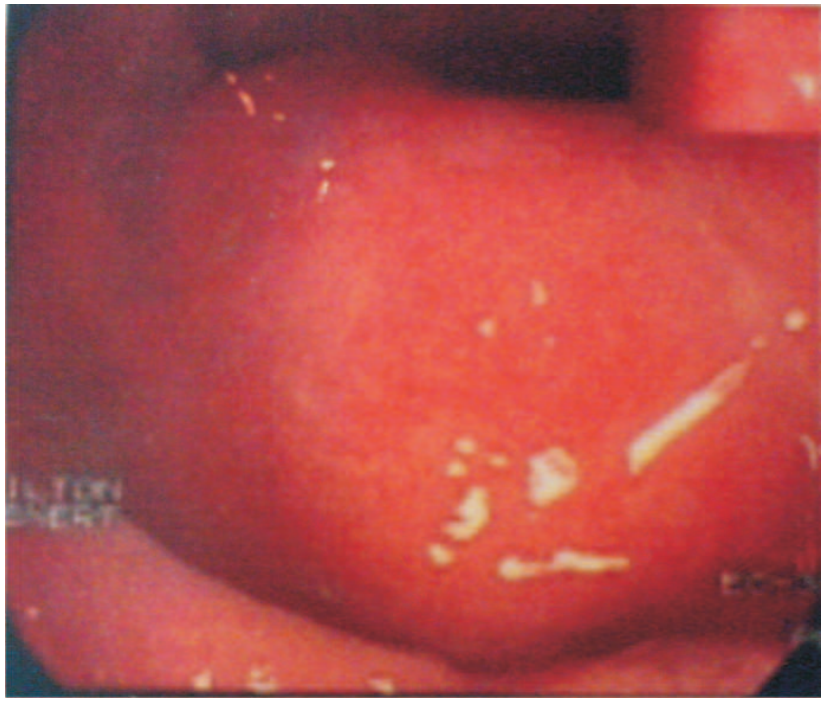

Figura 1 - Imagem colonoscópica do pólipo, indicando sua situação submucosa no reto.

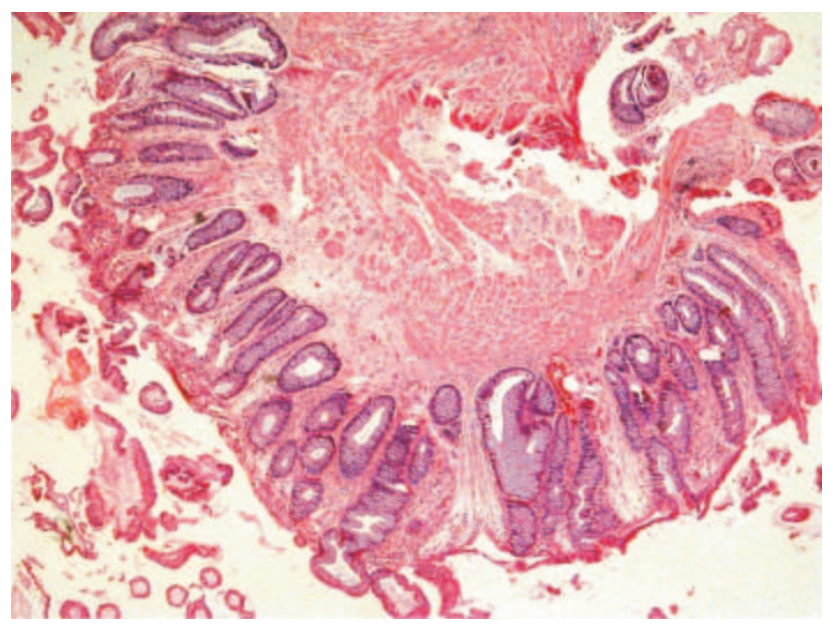

Figura 2 - Microscopia da biópsia, demonstrando aspecto adenomatoso viloso (pequeno aumento).

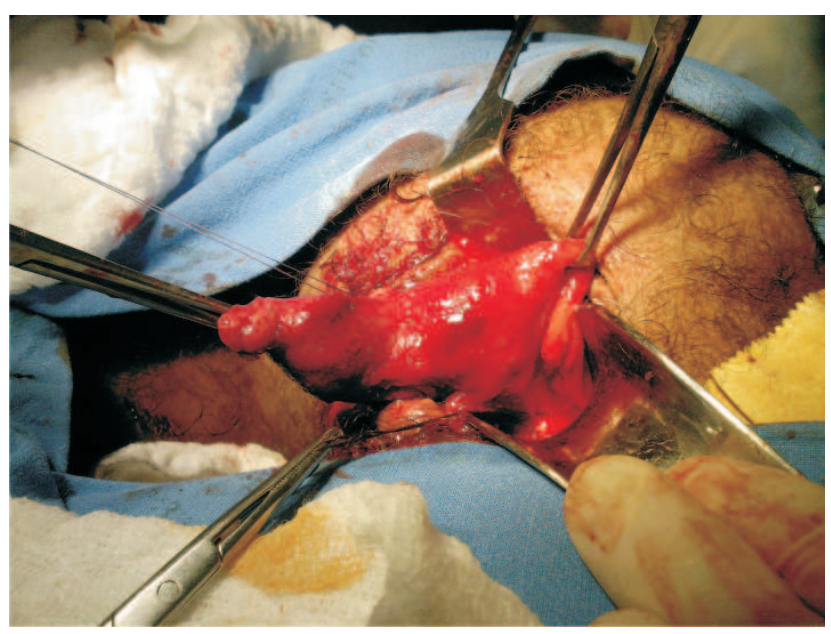

Figura 3 - Exérese transanal do pólipo. 


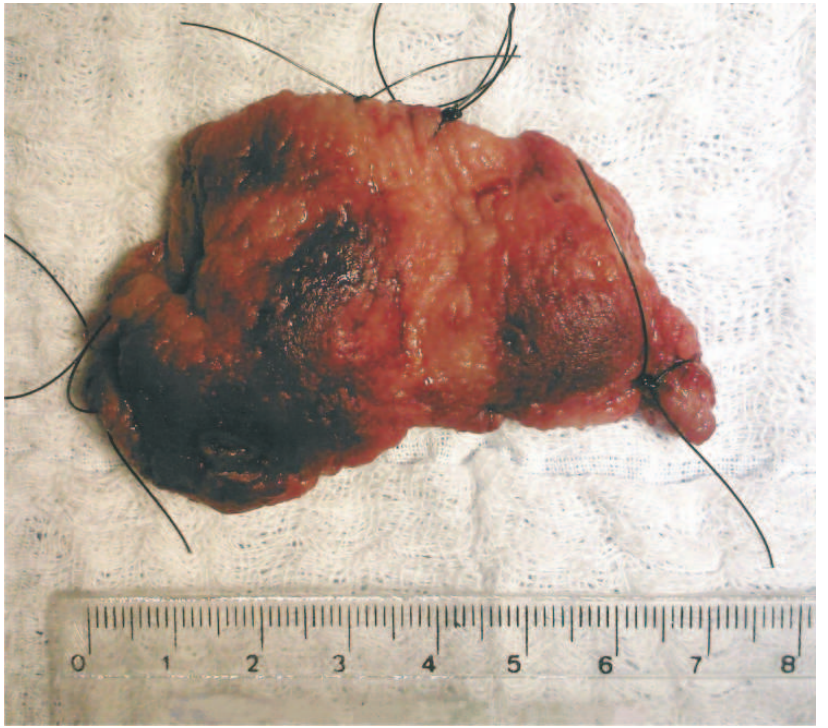

Figura 4-Aspecto macroscópico da peça cirúrgica.

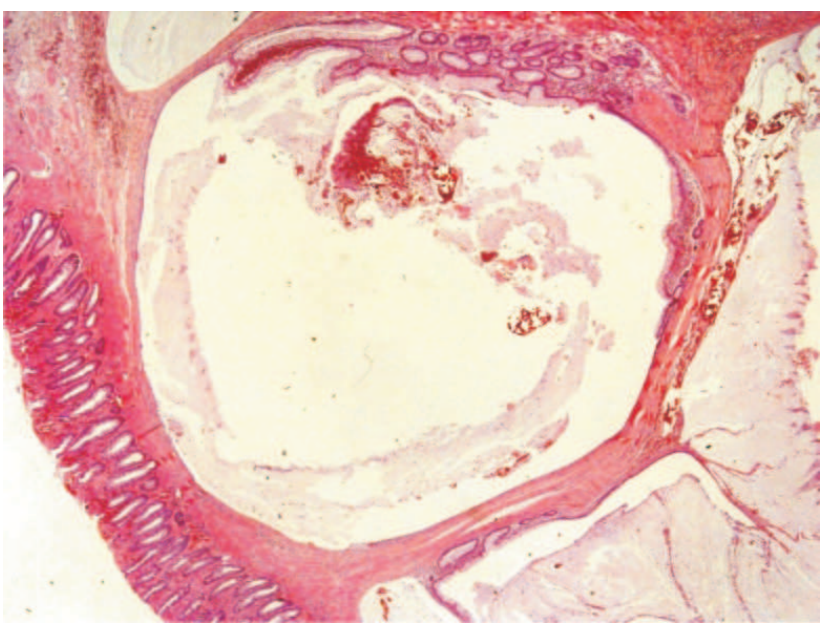

Figura 5 - Microscopia da peça cirúrgica, com visualização das lesões císticas na submucosa (pequeno aumento).

comumente associadas a doenças inflamatórias intestinais; podem ser polipoides ou papilares vilosas, ambas podendo apresentar ulcerações. A forma segmentar caracteriza-se por lesões - geralmente polipoides, em um ou mais segmentos colônicos (principalmente retossigmoide). A forma localizada, a mais frequente, apresenta geralmente lesão única, polipoide com ou sem ulceração e acomete o reto em sua parede anterior, entre 05 e $12 \mathrm{~cm}$ da margem anal ${ }^{(6)}$.

A apresentação clínica é variável, sendo relacionada à localização, à doença associada, ao número e ao aspecto macroscópico das lesões. Entre os principais sintomas estão a eliminação de sangue, muco, diarreia, tenesmo, dor, cólicas abdominais e dor retal.
Ao exame físico, o toque retal pode evidenciar nódulo ou ulceração ${ }^{(5-7,13)}$.

A avaliação endoscópica apresenta lesões polipoides, nódulos submucosos ou ulcerações. Os pólipos podem ser sésseis ou pediculados, únicos ou múltiplos, e estes quando confluentes podem formar uma massa de vários centímetros de diâmetro ${ }^{(1-4)}$.

A colite cística profunda apresenta diagnóstico diferencial extenso. As principais categorias inclu$\mathrm{em}$ as neoplasias benignas e malignas, doenças inflamatórias intestinais, infecções, colites medicamentosas e não-medicamentosas ${ }^{(10,13-16)}$.

Há apenas dois relatos de casos nos quais a colite cística profunda coexistia com adenocarcinoma na mesma lesão ${ }^{(4)}$. Acredita-se que ambos representam reações teciduais locais a algum estímulo / alteração, sendo que sua associação carece de estudos adicionais ${ }^{(6)}$.

O diagnóstico definitivo ocorre pela análise histopatológica, indicando suas características típicas e ausência de malignidade, sendo determinante para a conduta adequada ${ }^{(2,7)}$. Há relatos de pacientes submetidos a ressecções cirúrgicas amplas com base no diagnóstico equivocado de neoplasia maligna, bem como pacientes submetidos a tratamento conservador de adenocarcinoma mucinoso bem diferenciado ${ }^{(4)}$.

Histologicamente, situam-se na submucosa, espessando-a, eventualmente acomentendo a muscular própria, raramente a serosa do órgão. Os cistos são delimitados por células da mucosa colônica ou células epiteliais (escamosas ou colunares). É comum a comunicação do cisto com o lúmen intestinal, por aberturas através da muscular da mucosa. A mucosa geralmente apresenta-se edematosa, com tendência hipertrófica ou ainda atrófica. Úlceras superficiais são frequentes, bem como a infiltração de linfócitos, neutrófilos, plasmócitos, fibroblastos e células musculares lisas na lâmina própria, indicando sinais de inflamação tissular ${ }^{(1,7,8,12,13)}$.

A etiologia exata da colite cística profunda é controversa ${ }^{(5,7)}$. A hipótese congênita da lesão baseiase em três pontos: estudos em embriões evidenciaram cistos submucosos em diversos locais do trato gastrointestinal - embora a maioria das séries de autópsias não tenham encontrado os cistos; casos em pacientes pediátricos já foram relatados; há diversos casos descritos em pacientes portadores de síndrome de Peutz-Jeghers - doença autossômica que resulta na presença de hamartomas pelo tubo digestivo ${ }^{(6)}$. 
A origem adquirida é aventada pela freqüente concomitância com doenças que ocasionam irritação crônica intestinal, como a retocolite ulcerativa, doença de Crohn, pólipos adenomatosos, entre outros ${ }^{(7)}$. Os cistos também já foram encontrados em cirurgias gastrointestinais que expõem áreas a traumas persistentes, como colostomias ou anastomoses ${ }^{(6)}$. Existem diversos casos relatados de colite cística profunda pósradioterapia por neoplasia colorretal. Em modelos animais de inflamação intestinal induzida (expostos a radiação e pesticidas), as alterações encontradas remeteram à colite cística profunda ${ }^{(4)}$.

Acredita-se que as hipóteses etiológicas não são mutuamente excludentes. Existe a possibilidade de que nas autópsias os cistos teriam sido pequenos para serem visualizados ou ainda que tenham regredido. A colite cística poderia ser causada pela persistência dos cistos ou pela irritação e consequente inflamação da mucosa colorretal ${ }^{(7)}$.

Provavelmente, o mecanismo patogênico predominante é a isquemia ${ }^{(13)}$. Um estímulo inflamatório causaria a hipertrofia da parede de vênulas submucosas (ou endarterite obliterativa como após radioterapia), reduzindo o fluxo sanguíneo, ocasionando edema local e ulceração, por necrose mucosa focal. Tais lesões vasculares também levariam a defeitos na muscular da mucosa que seriam pontos de invaginação da mucosa quando da regeneração tissular, formando as áreas císticas ${ }^{(4)}$. Possivelmente a colite cística profunda também faz parte de um espectro patológico que inclui a síndrome da úlcera retal solitária e prolapso retal: a tração vascular originada pelo prolapso originaria alteração venular seguida de ulceração ${ }^{(16)}$.

As lesões pequenas e pouco sintomáticas são tratadas com aumento de fibras na dieta ou enemas com corticoesteroides ${ }^{(2)}$. Na persistência dos sintomas, indica-se a remoção cirúrgica da lesão ${ }^{(7)}$. Tornase fundamental a avaliação de causa subjacente, como doença inflamatória intestinal ou prolapso retal, cujo tratamento pode ocasionar a regressão da lesão cística ${ }^{(7,15)}$. O prognóstico é excelente, apresentando taxa de recorrência extremamente baixa ${ }^{(4)}$.

\section{CONCLUSÃO}

A colite cística profunda é doença benigna e rara, entretanto guarda íntima semelhança clínica e macroscópica com neoplasia maligna colorretal. Indica, ainda, frequente coexistência com outras desordens colorretais, como as doenças inflamatórias intestinais e prolapso retal. O tratamento consiste majoritariamente na remoção cirúrgica da lesão e terapêutica de doença colorretal de base.

ABSTRACT: Colitis cystica profunda comprises submucous mucus-filled cysts, located mainly in rectum and left colon. Its etiology is controversial, with about 200 cases reported in the literature. This disease is important clinically in that mimics colorectal malignancies. We report a case of colitis cystica profunda localized in rectum treated surgically, its follow-up and review of the literature.

Key words: Colitis, cysts, mucus, gastrointestinal hemorrage, rectum.

\section{REFERÊNCIAS}

1. Toro GC, Villaseca MH, Roa JCS. Colitis quística profunda posradioterapia. Caso clínico. Rev Med Chile 2007; 135:75963.

2. Kornprat $\mathrm{P}$, Langner C, Pfeifer J, Mischinger HJ. Colitis cystica profunda associated with rectal prolapse: report of a case. Int J Colorectal Dis 2007; 22:1555-6.

3. Hulsmans FJH, Tio TL, Reeders JWAJ, Tyigat GNJ. Transrectal US in the diagnosis of localized colitis cystica profunda. Radiol 1991; 181:201-33.

4. Guest CB, Reznick RK. Colitis cystica profunda-Review of the literature. Dis Colon Rectum 1989; 32: 983-8.
5. Alvarez RH, P, San Miguel G, Castro B. Colitis cystica profunda. Rev Esp Enferm Dig. 2008;100: 240-2.

6. Kim WH, Choe GY, Kim YI, Kim JP.Localized form of colitis cystica profunda- a case of occurrence in the descending colon. J Korean Med Sci. 1992;7:76-8.

7. Stuart M. Proctitis cystica profunda. Incidence, etiology, and treatment. Dis Colon Rectum 1984; 27: 153-6.

8. Herman AH, Nabseth DC. Colitis cystica profunda. Arch Surg 1973; 73: 337-41.

9. Ledesma-Medina J, Reid BS, Girdany BR. Colitis cystica profunda. Am J Roentgenol. 1978;131:529-30.

10. Laurent V, Corby S, Meyer-Bisch L, Ciprian-Corby S, Barbary C, Beot S, Bresler L, Régent D. Aspect IRM d'une 
pseudo tumeur rectale rare associée à des troubles dyschésiques: la "colitis cystica profunda". J Radiol 2007; 88:585-8.

11. Masahiro T, Nakamura T, Kawai H, Sawaki A, Mizuno N, Takahashi K, Yokoi T, Yatabe Y, et al. A case of colonic morule with colitis cystica profunda. Gastrointest Endosc 2007; 65:162-3.

12. Sztarkier I, Benharroch D, Walfisch S, Delgado J. Colitis cystica profunda and solitary rectal ulcer syndrome-polypoid variant: Two confusing clinical conditions. Eur J Intern Med 2006;17:578-9.

13. Levine DS. "Solitary" rectal ulcer syndrome. Are "solitary" rectal ulcer syndrome and "localized" colitis cystica profunda analogous syndromes caused by rectal prolapse? Gastroenterology 1987; 92: 243-53.
14. Madan A, Minocha A. First reported case of colitis cystica profunda in association with Chron's disease. Am J Gastroenterol 2002, 97:2472-3.

15. Dolar E, Kiyici M, Yilmazlar T, Gürel S, Nak SG, Gülten M. Colitis cystica profunda. Turk J Gastroenterol 2007; 18:206-7.

16. Wang F, Frisbie JH, Klein MA. Solitary rectal ulcer syndrome (colitis cystica profunda) in spinal cord injury patients: 3 case reports. Arch Phys Med Rahebil 2001; 82: 260-1.

\section{Endereço para correspondência:}

ILARIO FROEHNER JUNIOR

R. Dep. Antônio Edu Vieira, 1620, apto. 303 - F

Pantanal, Florianópolis - SC

CEP 88040-001

Telefones: (48) 9164-2198 / (48) 8431-1627

E-mail: froehnerjr@yahoo.com.br 